\title{
Nova Creatio: A Clinical Perspective on Rehabilitative Everyday Objects for People with Chronic Stroke
}

\begin{abstract}
Stroke affects 15 million people worldwide and is the third most likely cause of death. Unilateral impairments after a stroke affect the ability to carry out daily activities with the affected arm and hand. The preferential use of the less affected side can lead to a so-called learned nonuse. Although a number of assistive everyday objects are available to people with stroke, little is known about how everyday objects could be designed to be useful for a self-directed rehabilitation and how the user experience of stroke patients could be evaluated to design complex digital and/or physical systems. We developed a set of design criteria that other designers can use to create rehabilitative objects. We interviewed 12 stroke health professionals to investigate what the user experience in the rehabilitation process looks like, and which clinical criteria need to be considered in the context of designing rehabilitative objects. Interviews were analysed using thematic analysis. Results indicate that to encourage the use of the affected arm and hand in people who live at home and have reached the chronic stage of the recovery, self-efficacy needs to be in place. Furthermore, people must undergo a behaviour change in order to overcome the learned nonuse.
\end{abstract}

\section{Keywords}

Stroke Rehabilitation, Qualitative, Design Criteria, Learned Nonuse

\section{Introduction}

A stroke is a form of brain injury caused by lack of blood flow or oxygen delivery that leads to irreversible injuries to parts of the brain and can affect physiological as well as psychological abilities. A stroke affects approximately 15 million people annually worldwide, causing the deaths of one third, while another third survives with persistent disabilities [1]. Physical impairments commonly affect one side of the body in the form of hemiparesis, which is weakened muscles, or hemiplegia, which involves the paralysis of muscles [2]. Recovery from a stroke is a complex process that is likely caused by a learning-dependent process and a factor called spontaneous neurologic recovery [3]. The concept of spontaneous neurological recovery [4] is often referred to when explaining why improvements of motor impairments seem to plateau after the first weeks [5] in the upper limb within the first three months [6]. After this time, stroke survivors are likely to compensate for lost motor function of an arm with an enhanced movement of their less affected side, leading to a self-taught suppression of movement named the 'learned nonuse' of the affected arm [7]. Learned nonuse can be overcome by applying a physical restraint on the affected arm to evoke an initiation of use of the affected side while providing repetitive, contextualised training and behaviour change components. The intervention based on this principle is named constraint induced movement therapy (CIMT). 
Assistive technology [AT] is used after a stroke to compensate for the loss of functionality of the upper and lower limb and enable a safe discharge into the community. AT can be part of activities involving employment, mobility or communication $[8,9]$. Commonly provided products after discharge from hospital are used for bathing, walking, and home adaptations such as modification of stairs [10-12]. Products and technology that fall into the category of assistive products help compensate for the loss of functionality rather than contribute to the rehabilitation process.

While these objects may help stroke survivors carry out everyday activities again, we argue that they facilitate compensatory movement patterns and potentially 'learned nonuse', as they help avoid the use of the affected limb. We believe that everyday objects could be designed to facilitate the use of the stroke patients' affected side, therefore contributing to their recovery. It has further been reported that people suffering from complex regional pain syndrome can express behaviour patterns similar to learned nonuse after a stroke [13]. Designing for initiation of use of the affected limb for stroke rehabilitation is a highly complex human experience and specific design strategies can help designers to design digital or physical systems for it [14]. Our design strategies could potentially be beneficial for other clinical populations as well. Also, at this stage it is unclear what kind of design criteria such objects need to fulfil. We have developed a set of design criteria that other designers can refer to in order to design objects for a society that is becoming unavoidably older and more likely to survive a stroke and be affected by it. We conducted 12 semi-structured interviews with healthcare professionals to gain an understanding of clinical factors influencing the user experience of such novel designs and to develop a set of design criteria that other designers can refer to in the process. This will hopefully help designers in this challenging and complex system that involves non-typical physical abilities, cognitive challenges, and intended behaviour change.

\section{Methods}

\subsection{Semi-Structured Interview Content}

We used semi-structured interviews with stroke healthcare professionals to gain a deeper understanding of the rehabilitation process and strategies that can help to overcome learned nonuse. The primary questions in the interview guide were kept broad and open. Prompts that repeated the key concept were used during the interview to gain more in-depth information. The interview script outlined the main topics for the interview and started with questions about the participant's expertise. The flow of the interview influenced the order in which the remaining primary interview questions were asked. If participants did not elaborate on the concept of learned nonuse or had little involvement with chronic stroke survivors, additional follow-up questions were asked to ascertain if they were aware of the concept. Each interview was transcribed in full. For the interviews a set of main questions and follow-up questions were used:

- How is the rehabilitation process structured?

- Is the movement constrained during rehabilitation? If yes, in which form?

-Which kind of emotions are influencing the rehabilitation process?

-What kind of resources can people with stroke access?

- How would the ideal rehabilitation system look?

Follow up questions concerned the following points:

- How is feedback provided during the rehabilitation?

-What are the steps to set up goals for the rehabilitation?

- How are home-based assignments implemented in the process?

- How do you try to evoke an initiation of use of the affected arm and hand?

\subsection{Recruitment}

We interviewed health professionals who had worked with acute, subacute or chronic stroke patients in clinical practice or research context. The interviews took between 30 and 120 minutes (average 60 minutes). Non-probability snowball sampling [15] was chosen for this study, in which participants recommended further possible interviewees.

\subsection{Thematic Analysis}

The transcripts were analysed using thematic analysis in a theoretical or deductive form to identify, report, 
and analyse patterns and themes within the data [16]. Interviews were conducted by the first author. Initial codes and themes were generated by the first author and reviewed by the second and third authors.

\section{Results}

\subsection{Study Sample}

Twelve interviews were conducted with stroke therapists and stroke researchers. Eight interview partners were female and four were male. Three were occupational therapists; two worked as physiotherapists; one worked as a neuro physiotherapist; one worked as a professional therapist focusing on assessment post-stroke; one worked in the tertiary sector and had a background in musculoskeletal therapy; two worked as researchers focusing on stroke therapy; one worked as a scientist focusing on the recruitment of acute stroke patients for studies; and one was a supervisor of a clinic. Two therapists worked in Australia and ten worked in New Zealand.

\subsection{Design Criteria}

Based on analysis of the interviews, we developed a set of design criteria [17] that can be used in the design process to create everyday objects with a rehabilitative purpose.

\subsection{Themes}

The following section outlines details of the themes that were mentioned during the interviews. The section has been divided into firstly, factors that facilitate compensatory movement patterns after the stroke and consequently a neglect of use of the affected arm and hand and, secondly, the structure of rehabilitation interventions including CIMT that aim to minimise compensatory movement patterns and evoke an initiation of use among people with stroke.

\section{Factors Contributing to 'Learned Nonuse'. The} development of compensatory movement patterns and learned nonuse after a stroke is multifactorial and develops over time; see Figure 1. Four different factors were mentioned during the interviews that contribute to its development after stroke: physiological factors, the structure of current rehabilitation, individual behaviour, and social factors.

\begin{tabular}{|c|c|}
\hline Restrain & $\begin{array}{l}\text { The interaction with the objects needs } \\
\text { to restrain movement to evoke an } \\
\text { initiation of use and remind the user to } \\
\text { use the affected arm }\end{array}$ \\
\hline Contextualise & $\begin{array}{l}\text { The object needs to be used within an } \\
\text { activity of daily living }\end{array}$ \\
\hline Repetition & $\begin{array}{l}\text { The interaction with the object needs } \\
\text { to be repetitive }\end{array}$ \\
\hline Feedback & $\begin{array}{l}\text { Feedback needs to be positive, } \\
\text { immediate and quantitative }\end{array}$ \\
\hline Challenge & $\begin{array}{l}\text { The interaction with the object needs } \\
\text { to become progressively challenging }\end{array}$ \\
\hline Self-efficacy & $\begin{array}{l}\text { The interaction with the object needs } \\
\text { to contribute to developing self- } \\
\text { perceived self-efficacy of the user }\end{array}$ \\
\hline Usability & $\begin{array}{l}\text { The interaction with the object needs } \\
\text { to be achievable for a person with } \\
\text { motor impairments after a stroke }\end{array}$ \\
\hline Behaviour change & $\begin{array}{l}\text { A behaviour change component needs } \\
\text { to encourage the use of the affected } \\
\text { arm and hand in daily activities e.g., } \\
\text { behaviour change contract }\end{array}$ \\
\hline
\end{tabular}

Table 1. Design criteria

Physiological factors included factors such as cognitive impairments; apraxia, which causes difficulties in understanding the purpose of the rehabilitation; spasticity; fatigue; visual neglect; a shift of body awareness; and sensory deficits that can cause issues with proprioception and light touch. Once a person starts to compensate with an enhanced use of the less affected side, the muscles on the affected side start to decondition, causing a decline in muscle strength and muscle bulk.

What it tells us is that even a moderate level of impairment will promote nonuse. You have to be really, really good before you routinely use that hand and arm for everyday tasks and that is shocking. So the impairment scale is 66 out of 66 and we have found, and others have found, that before that if your score falls below around 55 you won't use that hand and arm. Your MAL [Motor Activity Log] score will drop below three for both amount and quality of use. Scientist 01 (Participant 05) 
Fig. 2. Factors influencing the development of learned nonuse based on interview results.

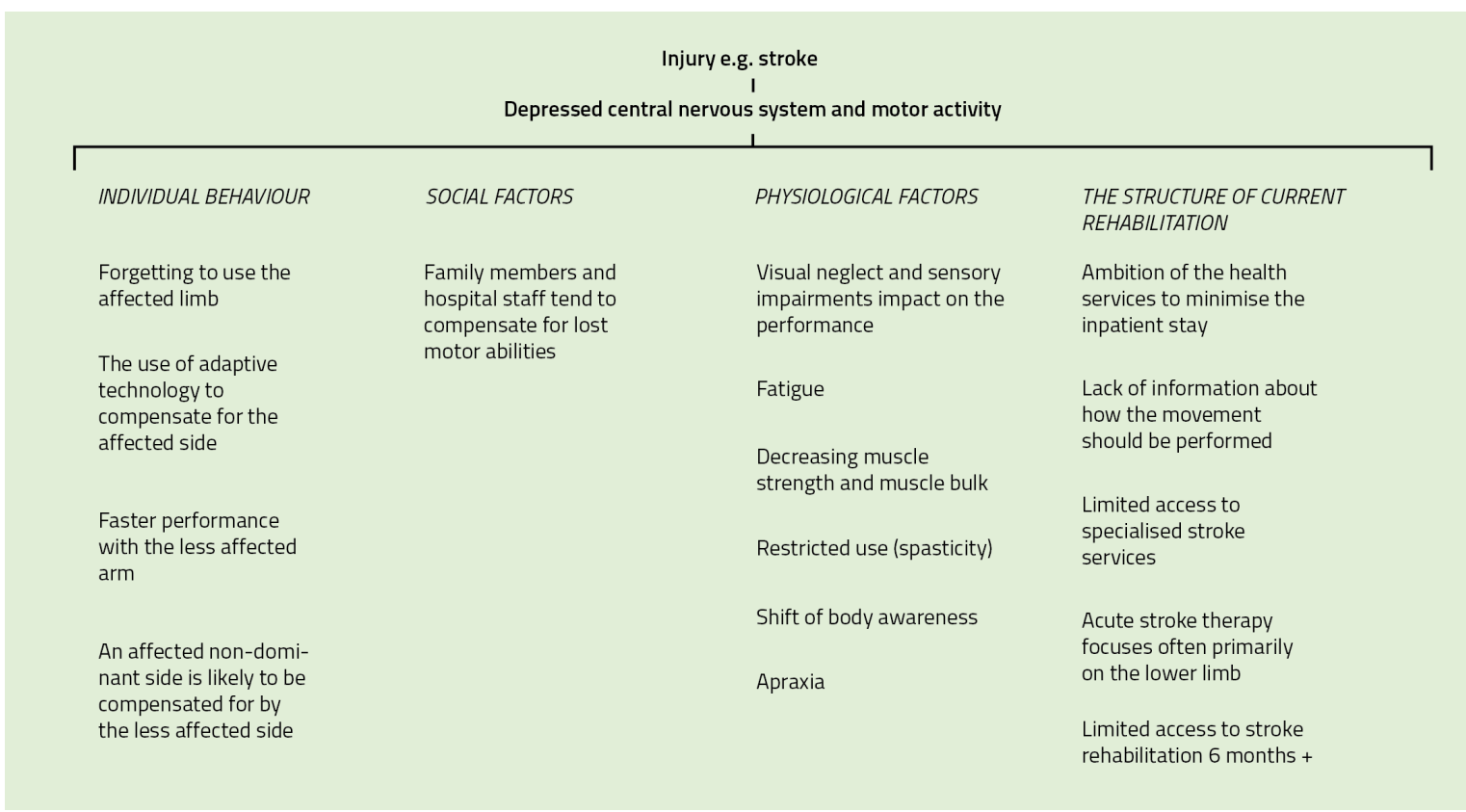

A focus in the acute and subacute rehabilitation process seems often to be on the lower limb, due to the stroke patients' wish to be able to walk again. This is also reinforced by the target setting of health services to minimise inpatient stay getting patients ambulatory for discharge. One result is that focus on the upper limb occurs relatively late in the rehabilitation process or not present at all.

It was further mentioned that people with a mild stroke and a rather high level of motor functionality are often confronted with statements telling them that they are not expected to get much better and that the focus is on more severe and acute patients. Historically, the focus on compensating for the loss of functionality occurs once a person has reached the chronic stage. The focus on compensation and use of assistive devices, often provided by occupational therapists, can further contribute to the development of learned nonuse by facilitating the use of the less affected side.

"They basically tell people when you are on a higher level: 'There is nothing you can do'; 'you are pretty good, so just be glad that you are as good as you are'; 'go home and don't worry about the things that you would like to do'; 'just thank god that you are alive and not too bad'. What is bad, I reckon. I think because I never ever met any stroke patient who told me that they are not too bad."

Occupational therapist (Participant 02)
People with stroke tend to neglect the affected side after failed attempts during the acute stage. The selftaught compensatory movement is reinforced through the easier and faster performance of the less affected side and simply forgetting to use the affected arm. It was further mentioned that the effects of 'learned nonuse' seem to be stronger in people with stroke who have reached a late chronic stage, that unsupervised training can reinforce compensatory movement patterns, and that there is an increased risk when the non-dominant hand is affected.

"People quite often quickly become one-handed especially when it is their non-dominant hand that is affected by the stroke." Physiotherapist (Participant 06)

Social factors contribute to the phenomenon as well. Relatives and occasionally hospital staff would try to make the life of the stroke survivors as easy as possible by doing tasks for them.

\section{Initiating the Use of the Affected Arm and}

Hand. In the following section the initial assessment, goal setting, feedback, emotions and elements of an effective rehabilitation intervention will be outlined. Figure 2 highlights which factors are part of a general rehabilitation right after the stroke. It additionally outlines themes mentioned in the context of learned nonuse. 


\begin{tabular}{|c|c|c|c|c|}
\hline \multicolumn{5}{|c|}{ Essential elements in the rehabilitation process } \\
\hline ASSESSMENT & GOAL SETTING & FEEDBACK & EMOTION & $\begin{array}{l}\text { CRUCIAL REHABILITATION } \\
\text { ELEMENTS }\end{array}$ \\
\hline $\begin{array}{l}\text { Determine motor deficits } \\
\text { Determine the amount of } \\
\text { learned nonuse } \\
\text { Therapist can understand } \\
\text { motivations, daily routine } \\
\text { and attitude of the } \\
\text { person with stroke } \\
\text { Baseline for outcome } \\
\text { measurements that can } \\
\text { be reflected back }\end{array}$ & $\begin{array}{l}\text { Often have given up trying } \\
\text { to use the arm at all } \\
\text { Goals need to be relevant } \\
\text { to the person, specific, } \\
\text { achievable, purposeful, } \\
\text { part of daily activities and } \\
\text { potentially competitive } \\
\text { Milestones are defined } \\
\text { that help during the } \\
\text { process to validate } \\
\text { improvements } \\
\text { Complex movements can } \\
\text { be broken down } \\
\text { Demonstrate the } \\
\text { correct movement } \\
\text { Include behaviour change } \\
\text { elements such as a } \\
\text { behaviour change contract }\end{array}$ & $\begin{array}{l}\text { Provide positive \& } \\
\text { instant feedback } \\
\text { Person needs to try to } \\
\text { perform the movement } \\
\text { before feedback is } \\
\text { provided } \\
\text { Improve movement } \\
\text { patterns and be a } \\
\text { motivational aid } \\
\text { Oral feedback as well as } \\
\text { 'hands-on' } \\
\text { Visual documentations } \\
\text { e.g. video recording } \\
\text { offers room for } \\
\text { discussion as to how to } \\
\text { improve the movement } \\
\text { Increase motivation to use } \\
\text { the affected arm } \\
\text { Increase self-efficacy }\end{array}$ & $\begin{array}{l}\text { Person has to develop a } \\
\text { feeling of responsibility } \\
\text { for their recovery process } \\
\text { Sadness, fear, } \\
\text { depression, grief, anger } \\
\text { or frustration about the } \\
\text { rehabilitation process } \\
\text { and funding } \\
\text { Have to accept the loss } \\
\text { of their previous role } \\
\text { Positive emotions were } \\
\text { mainly associated } \\
\text { positive rehabilitation } \\
\text { outcomes } \\
\text { Confronting situation } \\
\text { when they have to work } \\
\text { hard during the therapy }\end{array}$ & $\begin{array}{l}\text { Further practice and } \\
\text { reinforcement of using the } \\
\text { affected arm } \\
\text { Repeat the movement } \\
\text { Contextualise the } \\
\text { movement } \\
\text { Include family members in } \\
\text { the process } \\
\text { Provide information about } \\
\text { the structure and purpose } \\
\text { of the rehabilitation } \\
\text { Incorporate self-directed } \\
\text { training elements in a daily } \\
\text { routine } \\
\text { Take account of the } \\
\text { environment such as the } \\
\text { person's home }\end{array}$ \\
\hline
\end{tabular}

Fig. 3. Elements to initiate the use of the affected arm in clinical practice. Learned nonusespecific elements are highlighted in blue.
The initial assessment of the impairments helps the therapist determine motor deficits, to understand motivations, daily routine and attitudes of the person with stroke to set appropriate recovery goals. The assessment is further used as a baseline for outcome measurements that can be reflected back to the patient. In the process different assessment scales are often used. If people had reached the chronic stage of the stroke and received rehabilitation interventions such as CIMT, the therapist would determine the amount of learned nonuse by using assessment scales such as the motor activity log (MAL), which is a 30 -question interview guide to determine the amount and quality of daily use of the affected arm and hand.

Based on the assessment outcomes, appropriate goals are determined. Goals that are focused on during rehabilitation need to be relevant to the patient, specific, achievable, purposeful, part of daily activities and potentially competitive. The goals should be taskbased, rather than abstract ones such as 'I want to use my arm again'. It was further mentioned that they need to be contextualised.

The use of feasible, meaningful and specific goals for the process provides motivation in the often frustrating rehabilitation process. The person has to develop a feeling of responsibility for their process. Once the goals are determined, milestones are defined that help during the process to validate improvements. Complex movements can be broken down into individual components to then be mastered and learned as a sequence. Demonstrating the correct movement helps the patient understand how the movement needs to be performed effectively. Repetition of the correct movement is a core element of the process. Video recording is used to provide visual documentation and offer room for discussion on how to improve the movement.

The role of the therapist was described as a form of personal trainer that helps to reach the rehabilitation goals. They would provide motivational input in the form of feedback during the rehabilitation. Feedback is provided orally as well as in a 'hands-on' form. It should be provided instantly, appropriate for the person and task. Feedback is used to improve movement patterns as well as being a motivational aid.

So you want people to remain as positive and optimistic as possible but you have to be realistic so [...] it just needs to be realistic so saying something like 'Okay... I really see that you want get back to work but you are still in hospital right now'. So what steps do we need to take to get you there? 
"You need to do $x, y$, and $z$ but right now you just need to focus on the first part. To be able to get back to work you might need to be able to walk or you might need to be able to concentrate to complete a task for an hour or so."

Professional therapist (Participant 04)

In order to evoke an initiation of use of the upper limb in people with signs of learned nonuse the therapist has to provide sufficient motivational input and feedback to convince the patient that they should be trying to use the arm and that it is just the learned nonuse that impacts on their functional performance. When people with chronic stroke go back to see a therapist, they are often depressed and have given up trying to use the affected arm at all. When they are asked to work hard during the therapy session it can be experienced as an extremely confronting situation.

"You can't start these guys off trying to do more complicated things because they are doing nothing. So even if you think that they have some capacity [...]. The first couple of days with those people are always focused on trying to get them to just initiate the use of it and that can be anything really." Occupational therapist (Participant 02)

The emotions that the patient goes through can have a significant impact on the process. Most therapist mentioned mainly negative emotions such as sadness, fear, depression, grief, anger or frustration about the rehabilitation process and funding system. Patients must go through the process of accepting the loss of their previous role such as being the main provider for the family and accepting their new role and limitations post stroke. Rarely would someone see the stroke as a lifechanging event that transforms their life positively.

"We even have patients crying in the gym out of frustration because they are disappointed."

Supervisor of a student-run clinic (Participant 09)

Positive emotions were mainly associated with positive rehabilitation outcomes. The feeling of surprise after being able to perform movements was mentioned as evoking the wish to continue trying to use the affected arm in more activities once positive results have set in. It was emphasised that to overcome the signs of learned nonuse the person needs to go through behavioural changes. To secure an effective treatment, carers and family members are informed about and involved in the process. The use of a behaviour contract signed by the therapist and the person with stroke seems to be an effective tool to evoke a feeling of mutual responsibility. There are different techniques such as diaries or home practice sheets that help remind the patient to use the affected arm as often as possible.

The use of a physical constraint as part of CIMT to evoke an initiation of use was mentioned by a small number of our interview participants. The functional purpose is not primarily to restrict the movement, but to remind the patient to use the affected arm and limit the possibility of compensating with an enhanced use of the less affected side. It was emphasised that CIMT, which uses a physical constraint as part of the protocol, is a behaviour change intervention and that the physical constraint is just one element of the intervention.

\section{Discussion}

Assistive devices such as one-handed chopping boards and stroke cutlery are often provided to people who have experienced a stroke. These devices help to compensate for the lost motor abilities rather than contributing to the rehabilitation process once the person with stroke has returned home. However, devices such as the one-handed chopping board do not restrain movement of the affected arm and hand to contribute to an initiation of use, but enable the user to chop food one-handed. Furthermore, it does not contribute to a behaviour change to make sure that gains made in the process persist in the longterm. These are qualities commonly found in current rehabilitation devices. They demonstrate the lack of guidelines for designers to develop products and systems that properly address and evaluate the needs and complex situations that stroke patients face. This is not surprising, as designers also experience complexity when designing for stroke patients. Our findings will hopefully assist designers in this process by contributing to the body of knowledge in the discipline through strategies and concrete criteria. We conducted 12 interviews with stroke rehabilitation health professionals to develop an understanding of the user experience of people with stroke from multiple clinical perspectives. We developed a set of design criteria that 
can be used to develop objects with a rehabilitative character. Our results indicate that there is little focus on people who have reached the chronic stage of the stroke recovery and, due to financial and other resource limitations, the focus of current rehabilitation is often on the lower limb. It is therefore unclear if people who reach the chronic stage of the stroke have reached their full potential for recovery of use of their upper limb, or have started to express signs of learned nonuse. People with stroke have to face a constant struggle of accepting the new situation or waiting for improvements. Studies indicate that change can be experienced as necessary but associated at the same time with abandoning possible improvements by relying on technical aids, environmental improvements and other people [18].

Our study indicates several factors that contribute to the development of learned nonuse in people with stroke. The descriptions of physiological factors and individual behaviour contributing to learned nonuse correlate with its description in the literature as a self-taught behaviour that develops over time and is reinforced through unsuccessful motor attempts, failure, punishment and pain [19]. Additional personal and physiological factors mentioned in previous studies are: the extensive time to plan, initiate and complete a movement; fatigue; lack of confidence and control over the situation; pain; fear of negative consequences; and fear of becoming dependent on others $[18,20]$.

The findings of this study in terms of social factors correlate with previous findings [21]. Additional factors are social isolation caused by losing contact with friends and colleagues; not belonging to the work community; and attempts to hide the condition in order to appear 'normal' to others [20-22]. Another potential factor is gender. A study by Taub et al. [23] showed that female participants made greater gains on the 'Motor Activity Log' than males, with the possible explanation that the females were faced with a stronger expectation to use the affected arm to perform tasks again that are connected to traditional female roles such as housework and cooking.

Initiating the use of the affected arm and hand. The initiation of use of the affected arm and hand in the chronic stage of the stroke is crucial to overcoming the learned nonuse and decreased functional impairments. In the context of neuroplasticity, learned nonuse has been connected to the principle of 'use it or lose it' $[24,25]$, meaning that once compensatory movement patterns facilitate a neglect of the affected arm and hand potential underlying abilities to use the arm are lost. CIMT offers a detailed intervention protocol to overcome learned nonuse and has been part of our interview protocol. Few participants in the current study were familiar with the protocol. Participants who used CIMT mentioned that the protective safety mitt was preferred over the sling or cast to restrain movement and initiate use of the affected arm and hand. The mitt minimises current criticism regarding the patient's safety by only limiting the ability to use the fingers and hand in activities of daily living, but maintaining the ability to extend the arm in the case of a fall or to compensate for balance problems [26,27]. Therapists who used CIMT emphasised that the intervention aims to evoke a behaviour change rather than being repetitive task training and the constraint is not the main component, despite the name suggesting this [28]. Findings of this study show that the physical restraint reminds the patient of an extrinsic motivator [25] rather than limiting movement.

A number of factors were mentioned that seem to be unique in terms of initiating the use of the affected arm and hand and overcoming learned nonuse in the chronic stage of stroke. These are: assessment of the amount of learned nonuse; strategies to evoke the initiation of use based on assessed motor capabilities and motivational input; and the need to elicit a behaviour change. Feasible goals build the foundation to address learned nonuse successfully. The person with stroke is able to achieve the self-determined goals which improves self-efficacy [25]. Self-efficacy is the perceived competence to attain a specific level of performance in a given environment. It is the subjective cognition to carry out a particular behaviour and does not necessarily relate to the actual competence [29]. It seems to be essential to develop self-efficacy in order to choose to use the affected limb. However, little is known about it in the context of upper limb stroke rehabilitation due to a strong focus on the lower limb in most studies [25].

The mentioned elements of rehabilitation can be 
mapped to the elements of self-efficacy of mastery experiences, vicarious experiences, and verbal persuasion. However, the fourth source of self-efficacy, the physiological and emotional state [30], was not mentioned. The motivational input and keeping the patient engaged in the process of initiating use of the affected arm and hand was mentioned as a core element. Motivational input aims to convince the stroke survivor that they have the potential to use the arm and that it is just the self-taught behaviour that is stopping them from using it.

To address learned nonuse and evoke initiation of use, the rehabilitative object needs to address and increase self-efficacy beliefs. However, this is a complex process involving multiple factors. Self-efficacy beliefs rely on acquirable skills, increasing the belief of the individual that they can gain these skills, modelling the requisite skills, and defining activities in a way that make them achievable, as well as providing explicit feedback during the process [30]. A behaviour change component can help to secure gains made in the rehabilitation process in the long-term.

\section{Limitation}

The interview results need to be considered in light of the study sample. Two participants worked in Australia where the health system and stroke guidelines might be slightly different. Furthermore, most of the participants were located close to Wellington and Auckland which are two of New Zealand's largest cities. Access to stroke services might be different in provincial areas of the country and indeed internationally. Since none of the stroke therapists were located in a rural area, there might be a potential bias included in the results. We invited participants working in the private and public health sector. However, the majority of participants worked in private clinics and research contexts where people with stroke might be more encouraged to work on their motor deficits. A closer collaboration with local hospitals and hospital staff might potentially help to recruit participants who work with people with stroke who are less motivated.

It needs to be considered that the strategies focus on objects that can be used in the home environment as part of the rehabilitation process. Home-based stroke interventions were mentioned during the interviews as being a crucial element in the process. Nonetheless, there seems to be no consensus on one specific therapy approach. Furthermore, in New Zealand, access to stroke services for people with chronic stroke and mild impairments seems to be quite limited. One of the therapists mentioned that only highly motivated survivors with sufficient funding would try to improve motor impairments in the chronic stage, which indicates a lack of support for this stroke population.

People with stroke were not involved in this study and they could provide further insight about additional design criteria that influence the use of rehabilitative objects in the chronic stage of the stroke. We have taken account of this and applied for ethics with the Health and Disability Ethics Committees in New Zealand in order to involve people with stroke in the second stage of our research.

\section{References}

1. McKay, J., Mensah, GA. (2004). The atlas of heart disease and stroke. Geneva: World Health Organization.

2. Colman A.M. (2009). A dictionary of psychology [Internet]. Oxford: Oxford University Press. Retrieved Nov 12016 from: http://www.oxfordreference.com/views/BOOK_ SEARCH.html?book=t87

3. Langhorne, P., Bernhardt, J., Kwakkel, G. (2011). Stroke rehabilitation. The Lancet. 377(9778).

4. Gresham, GE. (1986). Stroke outcome research. Stroke. 17(3), 358-60.

5. Kwakkel, G., Kollen, B., Lindeman, E. (2004). Understanding the pattern of functional recovery after stroke: Facts and theories. Restorative Neurology and Neuroscience. 22(3-5), 281-99.

6. Zarahn, E., Alon, L., Ryan, S.L., Lazar, R.M., Vry, M-S., Weiller, C., et al. (2011). Prediction of motor recovery using initial impairment and fMRI 48 h Poststroke. Cerebral Cortex. 21(12), 2712-21.

7. Taub, E., Uswatte, G., Mark, V.W., Morris, D.M.M. (2006). The learned nonuse phenomenon: Implications for rehabilitation. Eura Medicophysica. 42(3), 241-56

8. Algurén, B., Lundgren-Nilsson, Å., Sunnerhagen, K.S. (2009) Facilitators and barriers of stroke survivors in the early poststroke phase. Disability and Rehabilitation. 31(19), 1584-91.

9. ICF Research Branch. (2013). Comprehensive ICF core set 
for stroke. Retrieved Jul 13 2017, from: https://www.icfresearch-branch.org/download/send/12-cardiovascularandres piratoryconditions/199-comprehensive-icf-core-set-stroke

10. de Barros, A.C., Duarte, C., Cruz, J.B. (2009). Designers and stroke research. Australasian Medical Journal. 10(1), 104-11.

11. Gosman-Hedström, G., Claesson, L., Blomstrand, C. (2002). Assistive devices in elderly people after stroke: A longitudinal, randomized study - The Göteborg 70+ Stroke Study. Scandinavian Journal of Occupational Therapy. 9(3), 109-18.

12. Sumathipala, K., Radcliffe, E., Sadler, E., Wolfe, C.D., McKevitt, C. (2012). Identifying the long-term needs of stroke survivors using the International Classification of Functioning, Disability and Health. Chronic Illness. 8(1), 31-44.

13. Punt, D.T., Cooper, L., Hey, M., Johnson, M.I. (2013). Neglectlike symptoms in complex regional pain syndrome: Learned nonuse by another name?. Pain. 154(2), 200-3.

14. Lemke, M., Rodríguez Ramírez, E., Robinson, B. (2017). Limited use only. How can the design of an everyday object help chronic stroke patients to overcome the learned nonuse of the upper limb?. The Design Journal. 28;20(sup1), S2397-2417.

15. Atkinson, R., Flint, J., (2011). Snowball Sampling. In: LewisBeck M, Bryman A, Futing Liao T, editors. The SAGE Encyclopedia of Social Science Research Methods. Thousand Oaks: Sage Publications, Inc. Retrieved Mar 232017 from: http://methods.sagepub.com/reference/the-sageencyclopedia-of-social-science-research-methods/n931.xml

16. Braun, V., Clarke, V. (2006). Using thematic analysis in psychology. Qualitative Research in Psychology. 3(2), 77-101.

17. Rodríguez Ramírez, E. (2017). A postgraduate thesis model for research through design based on design criteria. The International Journal of Designed Objects. 11(4), 11-27.

18. Wallenbert, I., Jonsson, H. (2005). Waiting to get better: A dilemma regarding habits in daily occupations after stroke. American Journal Occupational Therapy. 59(2), 218-24.

19. Taub, E., Morris, D.M. (2001). Constraint-induced movement therapy to enhance recovery after stroke. Current Atherosclerosis Reports. 3(4), 279-86.

20. Taule, T., Råheim, M. (2014). Life changed existentially: A qualitative study of experiences at 6-8 months after mild stroke. Disability and Rehabilitation. 36(25), 2107-19.

21. Bandura, A. (1997). Self-efficacy: The exercise of control. New York: W.H. Freeman.

22. Warner, G., Packer, T., Villeneuve, M., Audulv, A., Versnel, J. (2015). A systematic review of the effectiveness of stroke self-management programs for improving function and participation outcomes: Self-management programs for stroke survivors. Disability and Rehabilitation. 37(23), 2141-63.

23. Taub, E., Uswatte, G., Mark, V.W., Morris, D.M.M. (2006). The learned nonuse phenomenon: Implications for rehabilitation. Eura Medicophysica. 42(3), 241-56.

24. Cobley, C.S., Fisher, R.J., Chouliara, N., Kerr, M., Walker, M.F. (2013). A qualitative study exploring patients' and carers' experiences of early supported discharge services after stroke. Clinical Rehabilitation. 27(8), 750-7.

25. Dancause, N., Nadeau, S., Rossignol, S. (2015). Sensorimotor rehabilitation: At the crossroads of basic and clinical sciences. Amsterdam: Elsevier.

26. Taub, E., Crago, J.E., Uswatte, G. (1998). Constraint-induced movement therapy: A new approach to treatment in physical rehabilitation. Rehabilitation Psychology. 43(2):152-70.

27. Uswatte, G., Taub, E., Morris, D., Barman, J., Crago, J. (2006). Contribution of the shaping and restraint components of constraint-induced movement therapy to treatment outcome. NeuroRehabilitation. 21(2), 147-56.

28. Taub, E. (2012). The behavior-analytic origins of constraintinduced movement therapy: An example of behavioral neurorehabilitation. Behavioural Analysis. 35(2), 155-78.

29. Wood, J.P., Connelly, D.M., Maly, M.R. (2010). "Getting back to real living": A qualitative study of the process of community reintegration after stroke. Clinical Rehabilitation. 24(11), 1045-56.

30. Kleim, J.A., Jones, T.A. (2008). Principles of experiencedependent neural plasticity: Implications for rehabilitation after brain damage. Journal of Speech Language and Hearing Research. 51(1), 225-239. 